\title{
Impaired xanthine oxidoreductase (XOR)-mediated nitrite reductase activity possibly involved in the lack of antihypertensive effects of XOR inhibitors
}

Hypertension Research (2017) 40, 301; doi:10.1038/hr.2016.138; published online 13 October 2016

We read with interest the article by Kohagura et al. ${ }^{1}$ recently published in Hypertension Research about a historical cohort study among hypertensive patients with hyperuricemia who had recently started treatment with xanthine oxidoreductase (XOR) inhibitors. Enhanced formation of superoxide anion by XOR increases tissue concentrations of this free radical and other reactive oxygen species, thereby contributing to nitroso-redox imbalance in cardiovascular diseases, including hypertension. ${ }^{2}$ Indeed, increased vascular XOR activity or expression has been shown in different experimental models of hypertension. $^{3-5}$ However, in the present study, Kohagura et al. ${ }^{1}$ found no significant changes in blood pressure after treatment with XOR inhibitors. The investigators have suggested that these findings could be explained by the fact that the measurements obtained in the clinic may not be adequate to detect small changes in blood pressure in patients with controlled hypertension by using medications. We would like to add a different hypothesis to explain the lack of significant reduction in blood pressure after the treatment with XOR inhibitors.

In addition to its prooxidant activity, current evidence shows that XOR can convert nitrite anion into nitric oxide (NO), thus contributing to the beneficial effects of this vasodilator, particularly under certain circumstances. ${ }^{6}$ This property is very important, given that nitrate and nitrite are now considered as major sources of NO independent of enzymatic formation, constituting a complementary pathway known as the nitrate-nitrite-NO pathway. ${ }^{6}$ Importantly, diet represents the second major source of nitrite and nitrate in mammals. ${ }^{6}$ Dietary nitrate enters the circulation and is secreted into the oral cavity, where it is reduced to nitrite by oral commensal bacteria. Swallowed nitrite reaches the stomach and it is converted to $\mathrm{NO}$ and other bioactive nitrogen species under the acidic conditions of the gastric cavity. ${ }^{6}$ Nitrite is also absorbed from the intestine into the circulation and reduced to $\mathrm{NO}$ in blood and tissues by enzymes with nitrite reductase activity, including XOR. ${ }^{6}$ Indeed, it has been shown that vascular XOR contributes to the vasodilatory and hypotensive effects of nitrite in experimental models of hypertension and the use of XOR inhibitors can cancel this effect. ${ }^{4,5}$

Taking into account all these recent findings, it is possible that the use of allopurinol or febuxostat in this discussed article may have impaired nitrite-derived NO formation by XOR, thus preventing the antihypertensive effects of dietary nitrite and nitrate. Therefore, despite the very relevant findings regarding XOR inhibition on renal function, the nitrite reductase activity of XOR leading to NO production may be a protective antihypertensive mechanism that is lost with the use of XOR inhibitors.

\section{CONFLICT OF INTEREST}

The authors declare no conflict of interest.

Caio C Carvalho, Jose E Tanus-Santos and Gustavo H Oliveira-Paula

Department of Pharmacology, Ribeirao Preto Medical School, University of Sao Paulo, SP, Brazil

E-mail: gustavodepaula_88@yahoo.com.br

1 Kohagura K, Tana T, Higa A, Yamazato M, Ishida A, Nagahama K, Sakima A, Iseki K, Ohya Y. Effects of xanthine oxidase inhibitors on renal function and blood pressure in hypertensive patients with hyperuricemia. Hypertens Res 2016; 39: 593-597.

2 Hare JM. Nitroso-redox balance in the cardiovascular system. N Engl J Med 2004; 351: 2112-2114.

3 Amaral JH, Ferreira GC, Pinheiro LC, Montenegro MF, Tanus-Santos JE. Consistent antioxidant and antihypertensive effects of oral sodium nitrite in DOCA-salt hypertension. Redox Biol 2015; 5: 340-346.

4 Montenegro MF, Pinheiro LC, Amaral JH, Ferreira GC, Portella RL, Tanus-Santos JE. Vascular xanthine oxidoreductase contributes to the antihypertensive effects of sodium nitrite in L-NAME hypertension. Naunyn Schmiedebergs Arch Pharmacol 2014; 387: 591-598.

5 Oliveira-Paula GH, Pinheiro LC, Guimaraes DA, Tella SO, Blanco AL, Angelis CD, Schechter AN, Tanus-Santos JE. Tempol improves xanthine oxidoreductase-mediated vascular responses to nitrite in experimental renovascular hypertension. Redox Biol 2016; 8: 398-406.

6 Lundberg JO, Weitzberg E, Gladwin MT. The nitrate-nitrite-nitric oxide pathway in physiology and therapeutics. Nat Rev Drug Discov 2008; 7: 156-167. 